1 Universidade Federal dos Vales do Jequitinhonha e Mucuri (UFVJM),

Faculdade de Ciências Biológicas e da Saúde (FCBS) - Diamantina (MG), Brasil.

Orcid: https://orcid. org/0000-0002-2718-

2807

ronylascasas@gmail.com

2 Faculdade de Informática e Administração Paulista (Fiap) - São Paulo (SP),

Brasil.

Orcid: https://orcid org/0000-0002-7917-

3857

rbernal@usp.br

3 Universidade Federal de Minas Gerais (UFMG), Faculdade de Medicina

- Belo Horizonte (MG), Brasil.

Orcid: http://orcid. org/0000-0003-1366-

1732

alzira.o.jorge@gmail.com

4 Universidade Federal de Minas Gerais (UFMG), Faculdade de Medicina

- Belo Horizonte (MG),

Brasil.

Orcid: https://orcid. org/0000-0002-3577-

0721

elzammelo@hotmail.com

5 Universidade Federal de Minas Gerais (UFMG)

Escola de Enfermagem

- Belo Horizonte (MG),

Brasil.

Orcid: https://orcid

org/0000-0002-8214-

5734

dcmalta@uol.com.br

\section{Fatores associados à prática de Atividade Física na população brasileira - Vigitel 2013}

\author{
Factors associated with the practice of Physical Activity in the \\ Brazilian population - Vigitel 2013
}

Rony Carlos Rodrigues Las Casas', Regina Tomie Ivata Bernal2 , Alzira de Oliveira Jorge ${ }^{\mathbf{3}}$, Elza Machado de Melo ${ }^{4}$, Deborah Carvalho Malta 5

DOI: 10.1590/0103-11042018S410

RESUMO $\mathrm{O}$ artigo teve como objetivo analisar a associação entre a Atividade Física no Tempo Livre e as variáveis selecionadas nas capitais brasileiras. Foram analisadas informações do Sistema Nacional de Vigilância de Doenças Crônicas por Inquérito Telefônico, referentes ao ano de 2013, em uma amostra de 54 mil adultos nas capitais do Brasil. As Razões de Prevalências foram ajustadas segundo idade, sexo e escolaridade, utilizando-se regressão de Poisson com seus Intervalos de Confiança a 95\%. A prevalência da prática de Atividade Física no Tempo Livre foi de 33,8\% (IC95\%: 33,0-34,6), sendo maior entre homens (41,2\%) (IC95\%: 39,9-42,6) do que entre mulheres (27,4\%) (IC95\%: 26,5-28,3). Ser mais jovem, do sexo masculino, com maior escolaridade, da cor branca, possuir plano de saúde, não fumar e consumir o recomendado de frutas e hortaliças aumentaram a chance da prática da Atividade Física no Tempo Livre. Observou-se ainda que eutróficos e sobrepeso são mais ativos, obesos praticam menos Atividade Física. Conclui-se que a Atividade Física é benéfica à saúde, tornando-se importante investir em políticas públicas de promoção à saúde, visando criar oportunidades de inclusão social e equidade em saúde.

PALAVRAS-CHAVE Exercício. Atividades de lazer. Prevenção de doenças. Doenças crônicas não transmissíveis. Promoção da saúde.

ABSTRACT The article aims to analyze the association between Leisure Time Physical Activity and health variables in Brazilian capitals. Data from the National Surveillance System for Chronic Diseases by Telephone Survey, regarding the year 2013, were analyzed in a sample of 54,000 adults in Brazilian capitals. The Prevalence Ratios were adjusted according to age, gender and education by Poisson regression with their 95\% Confidence Intervals. The prevalence of Leisure Time Physical Activity was 33.8\% (95\% CI: 33.0-34.6), being higher among men 41.2\% (95\% CI: 39.9-42, 6) than among women 27.4\% (95\% CI: 26.5-28.3). Being younger, more educated, of white color, having a health insurance, not smoking, and consuming the recommended fruit and vegetables increased the chance of practicing Leisure Time Physical Activity. It was also observed that the eutrophic and the overweight are more active, the obese practice less Physical Activity. It is concluded that Physical Activity is beneficial to health, making it important to invest in public health promotion policies aimed at creating opportunities for social inclusion and equity in health.

KEYWORDS Exercise. Leisure activities. Disease prevention. Noncommunicable Diseases. Health promotion. 


\section{Introdução}

As Doenças Crônicas Não Transmissíveis (DCNT) são a principal causa de morbimortalidade no mundo ${ }^{1}$. Os fatores socioeconômicos, culturais, ambientais, os fatores não modificáveis (sexo, idade, herança genética) e os modificáveis (estilos de vida) são considerados determinantes sociais da saúde, das DCNT e da Atividade Física (AF) ${ }^{\mathbf{2}-4}$.

Os estilos de vida não saudáveis são advindos da vida moderna, sendo caracterizados pela maior utilização do tabaco, pelo aumento no consumo abusivo de bebida alcoólica, por um comportamento sedentário determinado pelo aumento do tempo de tela (televisão, jogos eletrônicos, telefones celulares) e por um comportamento alimentar inadequado, com predomínio da ingestão de alimentos ricos em gordura saturada, açúcar, sal, alimentos ultraprocessados; juntamente com a redução no consumo da dieta tradicional brasileira, mais saudável ${ }^{2}$.

Existem evidências científicas de que ações de promoção à saúde, cuidado e prevenção podem reduzir essas doençass,3. A prática regular da AF é um importante aliado no enfrentamento das DCNT, podendo ser entendida como qualquer movimento corporal realizado por músculos esqueléticos que gera um dispêndio energético maior do que os níveis de repouso realizados no lar, no trabalho, no deslocamento e nos períodos de tempo livre ${ }^{5}$.

A AF regular, principalmente no tempo livre, pode agregar inúmeros benefícios à saúde (melhora da resistência cardiorrespiratória, da composição corporal, da força, da resistência muscular e da flexibilidade) e às habilidades (agilidade, velocidade, potência, equilíbrio, coordenação e tempo de reação) ${ }^{6}$.

A inatividade física foi responsável pela morte de 3,2 milhões de pessoas ao ano, contribuindo para o crescimento de $20 \%$ a $30 \%$ na incidência de DCNT. Tal situação, além de acarretar aumento no investimento em saúde, aponta para a necessidade de ações de saúde pública, no sentido de um planejamento estratégico, com objetivos e metas claramente definidos, visando ao enfrentamento desse crescente problema de saúde ${ }^{1,7,8}$.

Segundo a Organização Mundial de Saúde (OMS), em adultos, a prática de 75 minutos semanais de AF intensa ou de 150 minutos semanais de AF moderada está relacionada com a contenção do aumento das DCNT e seus fatores associados, podendo, ainda, auxiliar no controle da pressão arterial e do diabetes mellitus; diminuir o colesterol sérico; as ocorrências de câncer de mama e de cólon, sendo relevante também para a redução do estresse e da depressão $0^{2,3,7,9}$.

É importante ressaltar que, além desses benefícios associados às dimensões biológicas e psicológicas, a AF propicia também interação e sociabilidade atuando, assim, na dimensão social10.

Na tese da colonização do mundo da vida de Habermas, a perda da interação e da sociabilidade produz individualismo metodológico na esfera privada e apatia na esfera pública, com a produção de importantes patologias sociais, como perda de sentido, anomia e psicopatologias"1. Portanto, a AF poderia representar importante estratégia de superação desses deficit, trazendo efeitos para a promoção de saúde das populações em seus vários ciclos de vida.

Existem poucos estudos que realizaram um monitoramento populacional dos níveis de AF que, em geral, além de ser um tema recente abordado na literatura, ficam concentrados nos países desenvolvidos?.

No Brasil, desde 2006, o Sistema Nacional de Vigilância de Doenças Crônicas por Inquérito Telefônico (Vigitel) vem coletando dados em todas as 26 capitais dos estados brasileiros e no Distrito Federal ${ }^{12}$. O objetivo do Vigitel é monitorar continuamente fatores de risco de DCNT na população adulta ( $\geq 18$ anos).

$\mathrm{O}$ monitoramento de indicadores da $\mathrm{AF}$ em âmbito populacional é essencial para subsidiar e apoiar políticas e ações de promoção à saúde e prevenção de doenças. Conhecê-los permite o enfrentamento das DCNT, além de subsidiar estudos que verifiquem a relação custo-efetividade incremental das boas práticas de $\mathrm{AF}^{\mathbf{1}, \mathbf{3}}$. Entre os indicadores monitorados no Vigitel, 
citam-se aqueles relativos à Atividade Física no Tempo Livre (AFTL), no deslocamento, inatividade física em quatro domínios e de tempo assistindo televisão ${ }^{\mathbf{1 2}}$.

O objetivo do presente estudo foi identificar associações da prática da AFTL com características sociodemográficas, estilo de vida, Índice de Massa Corporal (IMC), doenças crônicas autorreferidas e avaliação do estado de saúde.

\section{Métodos}

Trata-se de um estudo transversal, contendo análise das informações obtidas pelo Vigitel, realizado em 2013 com a população adulta ( $\geq 18$ anos de idade) nas 26 capitais dos estados brasileiros e no Distrito Federal. O Vigitel utiliza amostras probabilísticas da população adulta nas capitais a partir do cadastro das linhas de telefone fixo das cidades. Foram realizadas cerca de $54 \mathrm{mil}$ entrevistas, sendo 2 mil em cada capital e no Distrito Federal, das linhas residenciais ativas elegíveis. Pesos pós-estratificação foram utilizados para cada indivíduo entrevistado pelo Vigitel, de forma a padronizar a composição sociodemográfica estimada para a população de adultos de cada capital e do Distrito Federal. Esses pesos atribuídos visam homogeneizar a composição sociodemográfica da população total e da população com telefone nas variáveis sexo, faixa etária e nível de instrução.

A partir de 2012, os pesos pós-estratificação de cada indivíduo da amostra Vigitel foram calculados pelo método Rake, utilizando rotina específica do programa Stata Statistical Software ${ }^{\mathbf{1 3}}$. Esse método utiliza procedimentos interativos com sucessivas comparações entre estimativas da distribuição de cada variável sociodemográfica na amostra Vigitel e na população total da cidade.

O questionário do Vigitel engloba, aproximadamente, 94 questões divididas em módulos, como características demográficas e socioeconômicas dos indivíduos; padrão de alimentação e de AF; peso e altura referidos; morbidade referida; entre outros ${ }^{12}$.
O desfecho analisado (variável dependente) foi à prática de AFTL. Esse indicador foi estimado a partir das questões: 'Nos últimos três meses, $\mathrm{o}$ (a) Sr. (a) praticou algum tipo de exercício físico ou esporte?'; 'Qual o tipo principal de exercício físico ou esporte que o (a) Sr. (a) praticou?'; 'O (a) Sr. (a) pratica o exercício pelo menos uma vez por semana?'; 'Quantos dias por semana o (a) Sr. (a) costuma praticar exercício físico ou esporte?' e 'No dia que o (a) Sr. (a) pratica exercício ou esporte, quanto tempo durou esta atividade?'.

O cálculo considerou o número de indivíduos que praticam pelo menos 150 minutos semanais de $\mathrm{AF}$ de intensidade leve ou moderada ou, pelo menos, 75 minutos semanais de AF de intensidade vigorosa/número de indivíduos entrevistados, independentemente do número mínimo de dias na semana para a prática da $\mathrm{AF}^{7}$.

Caminhada, caminhada em esteira, musculação, hidroginástica, ginástica em geral, natação, artes marciais, ciclismo e voleibol foram classificados como práticas de intensidade leve ou moderada. Corrida, corrida em esteira, ginástica aeróbica, futebol, basquetebol e tênis foram classificados como práticas de intensidade vigorosa ${ }^{12}$.

Foram realizadas análise bivariada e cálculo de prevalência da prática de AFTL com Intervalo de Confiança de $95 \%\left(\mathrm{IC}_{95 \%}\right)$. Calculou-se a Razão de Prevalência (RP) pelo método de regressão de Poisson com variância robusta, brutas e ajustadas segundo idade, sexo e escolaridade para a população adulta total e por idade e escolaridade em cada categoria de sexo. $\mathrm{O}$ ajuste foi realizado com intuito de controlar a influência dessas variáveis nas estimativas.

As variáveis explicativas estudadas foram: características sociodemográficas (sexo, idade, escolaridade, raça/cor e ter plano de saúde), fatores de risco para as DCNT (tabaco, IMC e consumo abusivo de bebida alcoólica), fatores de proteção para as DCNT (consumo recomendado de frutas e hortaliças - cinco ou mais porções diárias e por semana), doença crônica autorreferida (diabetes, hipertensão, colesterol elevado e doenças crônicas associadas).

Para o processamento dos dados e análises, utilizou-se o aplicativo Stata versão 12.114. Este 
estudo foi aprovado pela Comissão Nacional de Ética em Pesquisa (Conep) em seres humanos, sob os pareceres 13.081 de 2008 e 355.590 de 2013.

\section{Resultados}

No conjunto das 27 capitais do País, a prevalência da prática de AFTL equivalente a 150 minutos de atividade moderada ou 75 minutos de atividade intensa por semana foi de $33,8 \%$ ( $\mathrm{IC}_{95 \%}: 33,0-34,6$ ), sendo maior entre homens $(41,2 \%)\left(\mathrm{IC}_{95 \%}: 39,9-42,6\right)$ do que entre mulheres $(27,4 \%)\left(\mathrm{IC}_{95 \%}: 26,5-28,3\right)$. Em todas as faixas etárias, a frequência dessa condição foi maior entre os homens. No entanto, em ambos os sexos, observou-se redução da prática de AFTL com o aumento da idade, sendo 49,7\% $\left(\mathrm{IC}_{95 \%}: 47,41-51,99\right)$ para 18 a 24 anos; $39,28 \%$ ( $\left.\mathrm{IC}_{95 \%}: 37,39-41,17\right)$ entre 25 e 34 anos e chegando a $22,34 \%\left(\mathrm{IC}_{95 \%}: 20,71-23,96\right)$ para 65 anos e mais. Essa redução ocorreu em ambos os sexos, sendo mais acentuada entre os homens.

Os níveis de AFTL aumentaram com o nível de escolaridade em ambos os sexos, sendo que entre 0 e 8 anos de escolaridade foi de $22,02 \%\left(\mathrm{IC}_{95 \%}: 20,76-23,28\right)$; de 9 a 11 anos de escolaridade foi de $37,18 \%\left(\mathrm{IC}_{95 \%}: 35,89-38,46\right)$ e 12 anos e mais de escolaridade foi de $45,41 \%$ ( $\mathrm{IC}_{95 \%}$ : 43,84-46,98). Mulheres brancas foram mais ativas $(30,48 \%)\left(\mathrm{IC}_{95 \%}: 28,93-32,03\right)$, em comparação com mulheres pretas e pardas. Populações com plano de saúde são mais ativas, sendo que a frequência foi de 39,27\% ( IC $\left._{95 \%}: 38,11-40,44\right)$ em comparação aos que não possuem plano, que foi de $28,81 \%\left(\mathrm{IC}_{95 \%}\right.$ : 27,72-29,9). O não fumante é mais ativo, comparado com o ex-fumante e o fumante, sendo que as frequências foram, respectivamente, $36,37 \%\left(\mathrm{IC}_{95 \%}: 35,38-37,36\right) ; 30,12 \%\left(\mathrm{IC}_{95 \%}\right.$ : 28,51-31,73) e $25,4 \%\left(\mathrm{IC}_{95 \%}: 22,9-27,9\right)$ (tabela 1).

Pessoas eutróficas ou com sobrepeso são mais ativas, com, respectivamente, $34,59 \%$ ( $\mathrm{IC}_{95 \%}$ : $33,42-35,75)$ e $35,97 \%$ ( $\left.\operatorname{IC}_{95 \%}: 34,57-37,36\right)$, do que as pessoas obesas $27,27 \%\left(\mathrm{IC}_{95 \%}: 25,48-29,05\right)$. Indivíduos que consomem o recomendado, como cinco ou mais porções diárias ou dias por semana de frutas e hortaliças, são mais ativos - 38,34\% $\left(\mathrm{IC}_{95 \%}: 37,15-39,53\right)$ e $43,17 \%$ ( $\left.\mathrm{IC}_{95 \%}: 41,49-44,85\right)$ respectivamente - do que aqueles que não consomem. Ao contrário, aqueles que apresentam consumo abusivo de bebida alcoólica foram mais ativos $(41,5 \%)\left(\mathrm{IC}_{95 \%}: 39,33-43,68\right)$. As pessoas que possuem mais doenças crônicas, hipertensão, diabetes e colesterol elevado, em geral, praticam menos AFTL.

Tabela 1. Prevalência de adultos (+ de 18 anos) que praticavam atividade física suficiente no tempo livre segundo sexo*. Conjunto da população das capitais dos estados brasileiros e Distrito Federal. Vigitel, 2013

\begin{tabular}{|c|c|c|c|c|c|c|c|c|c|}
\hline \multirow[t]{2}{*}{ Variáveis } & \multicolumn{3}{|c|}{ Total } & \multicolumn{3}{|c|}{ Masculino } & \multicolumn{3}{|c|}{ Feminino } \\
\hline & $\%$ & & $\mathrm{IC}_{95} \%$ & $\%$ & & $\mathrm{IC}_{95} \%$ & $\%$ & & $\mathrm{IC}_{95} \%$ \\
\hline Total & 33,8 & 33 & 34,6 & 41,2 & 39,9 & 42,6 & 27,4 & 26,5 & 28,3 \\
\hline \multicolumn{10}{|c|}{ Idade (anos) } \\
\hline 18 a 24 & 49,70 & 47,41 & 51,99 & 63,55 & 60,42 & 66,67 & 34,51 & 31,54 & 37,49 \\
\hline 25 a 34 & 39,28 & 37,39 & 41,17 & 48,05 & 45,12 & 50,98 & 30,66 & 28,32 & 33,00 \\
\hline 35 a 44 & 29,58 & 27,90 & 31,26 & 34,20 & 31,30 & 37,11 & 25,86 & 23,92 & 27,79 \\
\hline 45 a 54 & 27,34 & 25,70 & 28,97 & 29,48 & 26,75 & 32,22 & 25,63 & 23,65 & 27,61 \\
\hline 55 a 64 & 26,64 & 24,86 & 28,42 & 28,47 & 25,48 & 31,47 & 25,35 & 23,17 & 27,54 \\
\hline 65 e mais & 22,34 & 20,71 & 23,96 & 26,23 & 23,33 & 29,13 & 19,87 & 17,92 & 21,81 \\
\hline \multicolumn{10}{|c|}{ Escolaridade (anos) } \\
\hline 0 a 8 & 22,02 & 20,76 & 23,28 & 25,10 & 23,00 & 27,20 & 19,31 & 17,84 & 20,79 \\
\hline 9 a 11 & 37,18 & 35,89 & 38,46 & 48,40 & 46,36 & 50,44 & 27,26 & 25,77 & 28,74 \\
\hline 12 e mais & 45,41 & 43,84 & 46,98 & 54,30 & 51,79 & 56,82 & 38,44 & 36,50 & 40,38 \\
\hline
\end{tabular}


Tabela 1. (cont.)

\begin{tabular}{|c|c|c|c|c|c|c|c|c|c|}
\hline \multicolumn{10}{|c|}{ Raça/cora } \\
\hline Branca & 35,48 & 34,20 & 36,75 & 41,75 & 39,64 & 43,87 & 30,48 & 28,93 & 32,03 \\
\hline Preta & 32,11 & 29,58 & 34,64 & 41,78 & 37,53 & 46,03 & 23,03 & 20,34 & 25,73 \\
\hline Parda & 33,99 & 32,65 & 35,32 & 43,63 & 41,40 & 45,86 & 25,57 & 24,07 & 27,06 \\
\hline \multicolumn{10}{|c|}{ Plano de saúde } \\
\hline Sim & 39,27 & 38,11 & 40,44 & 47,12 & 45,20 & 49,03 & 32,91 & 31,50 & 34,32 \\
\hline Não & 28,81 & 27,72 & 29,90 & 36,18 & 34,35 & 38,01 & 22,22 & 20,98 & 23,45 \\
\hline \multicolumn{10}{|c|}{ Tabagismo } \\
\hline Não fumante & 36,37 & 35,38 & 37,36 & 47,01 & 45,28 & 48,74 & 28,85 & 27,73 & 29,96 \\
\hline Ex-fumante & 30,12 & 28,51 & 31,73 & 33,58 & 31,20 & 35,97 & 26,12 & 24,01 & 28,23 \\
\hline Fumante & 25,40 & 22,90 & 27,90 & 30,59 & 26,95 & 34,23 & 17,95 & 14,91 & 20,98 \\
\hline \multicolumn{10}{|c|}{ IMC } \\
\hline Normal & 34,59 & 33,42 & 35,75 & 42,62 & 40,59 & 44,65 & 28,68 & 27,34 & 30,02 \\
\hline Sobrepeso & 35,97 & 34,57 & 37,36 & 43,17 & 41,01 & 45,32 & 28,29 & 26,59 & 29,98 \\
\hline Obesidade & 27,27 & 25,48 & 29,05 & 33,41 & 30,42 & 36,40 & 22,03 & 19,95 & 24,11 \\
\hline \multicolumn{10}{|c|}{ Consumo recomendado de frutas e hortaliças ( 5 ou mais porções diárias) } \\
\hline Sim & 38,34 & 37,15 & 39,53 & 46,13 & 44,15 & 48,10 & 31,88 & 30,47 & 33,29 \\
\hline Não & 29,38 & 28,30 & 30,45 & 36,65 & 34,87 & 38,43 & 22,98 & 21,73 & 24,23 \\
\hline \multicolumn{10}{|c|}{ Consumo recomendado de frutas e hortaliças ( 5 ou mais dias por semana) } \\
\hline Sim & 43,17 & 41,49 & 44,85 & 51,37 & 48,32 & 54,43 & 38,21 & 36,26 & 40,15 \\
\hline Não & 30,86 & 29,95 & 31,76 & 38,78 & 37,31 & 40,25 & 23,35 & 22,29 & 24,40 \\
\hline \multicolumn{10}{|c|}{ Consumo abusivo de bebidas alcoólicas } \\
\hline Não & 32,24 & 31,39 & 33,10 & 39,91 & 38,39 & 41,43 & 26,75 & 25,77 & 27,72 \\
\hline $\operatorname{Sim}$ & 41,50 & 39,33 & 43,68 & 45,28 & 42,54 & 48,02 & 33,45 & 30,04 & 36,87 \\
\hline \multicolumn{10}{|c|}{ Hipertensão } \\
\hline Não & 36,47 & 35,51 & 37,43 & 44,12 & 42,57 & 45,67 & 29,50 & 28,35 & 30,65 \\
\hline Sim & 25,23 & 23,88 & 26,59 & 30,57 & 28,12 & 33,02 & 21,52 & 19,99 & 23,04 \\
\hline \multicolumn{10}{|c|}{ Diabetes } \\
\hline Não & 34,51 & 33,67 & 35,35 & 42,13 & 40,75 & 43,52 & 27,96 & 26,97 & 28,94 \\
\hline $\operatorname{Sim}$ & 23,59 & 20,97 & 26,20 & 27,97 & 23,36 & 32,58 & 20,20 & 17,24 & 23,16 \\
\hline \multicolumn{10}{|c|}{ Colesterol } \\
\hline Não & 34,91 & 33,98 & 35,83 & 42,60 & 41,11 & 44,09 & 27,85 & 26,74 & 28,95 \\
\hline Sim & 29,26 & 27,70 & 30,82 & 34,54 & 31,66 & 37,41 & 25,89 & 24,12 & 27,65 \\
\hline \multicolumn{10}{|c|}{ № de doenças crônicas } \\
\hline 0 & 37,10 & 36,02 & 38,17 & 45,16 & 43,45 & 46,86 & 29,49 & 28,18 & 30,79 \\
\hline 1 & 30,04 & 28,61 & 31,46 & 35,30 & 32,84 & 37,77 & 26,02 & 24,36 & 27,67 \\
\hline 2 ou 3 & 23,85 & 21,96 & 25,75 & 28,56 & 24,96 & 32,16 & 20,90 & 18,80 & 22,99 \\
\hline
\end{tabular}

Fonte: Elaboração própria, com base em dados da Vigitel, 2013.

*A prevalência da prática de atividade física no tempo livre equivale a 150 minutos de atividade moderada ou 75 minutos de atividade intensa por semana.

a Excluídas as categorias cor da pele amarela, vermelha, não sabe e não quis informar.

Nota: percentual ponderado para ajustar a distribuição sociodemográfica da amostra Vigitel à distribuição da população adulta de cada cidade projetada para o ano de 2012. 
As razões de prevalência apresentadas na tabela 2 foram ajustadas por idade, sexo e escolaridade. Descrevem a magnitude das associações da prática da AFTL às variáveis explicativas. Em relação à idade, tomando-se como referência a faixa etária de 18 a 24 anos, verificou-se uma redução da prática de AFTL com o aumento da idade. Os indivíduos entre 25 e 34 anos apresentaram $\mathrm{RP}$ de $0,76\left(\mathrm{IC}_{95 \%}\right.$ : $0,72-0,81)$ reduzindo com a idade de 65 anos ou mais para $\mathrm{RP}$ de $0,6\left(\mathrm{IC}_{95 \%}: 0,55-0,65\right)$. A prática de AFTL aumentou com a escolaridade. Comparados a indivíduos com até 8 anos de escolaridade, aqueles com 9 a 11 anos e 12 anos ou mais apresentaram, respectivamente, a RP de $1,48\left(\mathrm{IC}_{95 \%}\right.$ : 1,37-1,58) e 1,83 ( $\mathrm{IC}_{95 \%}$ : 1,7-1,96). Comparadas com as de cor branca, as mulheres da cor parda e preta são menos ativas, com RP de $0,87\left(\mathrm{IC}_{95 \%}: 0,81-0,94\right)$ e $0,8\left(\mathrm{IC}_{95 \%}: 0,7-0,91\right)$ respectivamente. Os homens que não possuem plano de saúde são mais ativos, com RP de 1,1 ( $\mathrm{IC}_{95 \%}$ : 1,04-1,19); e as mulheres sem planos de saúde são menos ativas, com RP de 0,77 $\left(\mathrm{IC}_{95 \%}: 0,72-0,84\right)$. Os fumantes de ambos os sexos praticam menos AFTL, com RP de 0,74 ( $\mathrm{IC}_{95 \%}$ : 0,67-0,81) (tabela 2).

Comparadas com as pessoas eutróficas, aquelas em sobrepeso são mais ativas, com $\mathrm{RP}$ de 1,08 ( $\mathrm{IC}_{95 \%}$ : 1,03-1,14); e pessoas com obesidade, menos ativas, com RP de 0,88 ( $\left.\mathrm{IC}_{95 \%}: 0,82-0,95\right)$. Essa relação se mantém para homens e mulheres. Em ambos os sexos, a prática de AFTL é maior naqueles que consomem frutas e hortaliças. Mulheres que consomem abusivamente bebida alcoólica praticam mais AFTL, com RP de 1,13 ( IC $\left._{95 \%}: 1,02-1,25\right)$. Não foram observadas diferenças na prática de AFTL para indivíduos com doenças crônicas (hipertensão, diabetes, hipercolesterolemia e número de doenças crônicas).

Tabela 2. Intervalo de Confiança (95\%) para razões de prevalência de adultos (+ de 18 anos) que praticavam atividade física suficiente no tempo livre segundo sexo. Conjunto da população das capitais dos estados brasileiros e Distrito Federal. Vigitel, 2013

\begin{tabular}{|c|c|c|c|c|c|c|c|c|c|}
\hline \multirow[t]{2}{*}{ Variáveis } & \multicolumn{3}{|c|}{ Total } & \multicolumn{3}{|c|}{ Masculino } & \multicolumn{3}{|c|}{ Feminino } \\
\hline & $\mathrm{RP}^{\star}$ & & IC95\% & $R^{\star \star \star}$ & & IC95\% & $R P^{\star \star \star}$ & & IC95\% \\
\hline \multicolumn{10}{|c|}{ Idade (anos) } \\
\hline 18 а 24 & 1,00 & & & 1,00 & & & 1,00 & & \\
\hline 25 a 34 & 0,76 & 0,72 & 0,81 & 0,72 & 0,66 & 0,78 & 0,86 & 0,77 & 0,96 \\
\hline 35 a 44 & 0,65 & 0,60 & 0,69 & 0,58 & 0,52 & 0,63 & 0,78 & 0,70 & 0,87 \\
\hline 45 a 54 & 0,62 & 0,57 & 0,66 & 0,50 & 0,45 & 0,55 & 0,82 & 0,73 & 0,91 \\
\hline 55 a 64 & 0,64 & 0,59 & 0,69 & 0,50 & 0,45 & 0,56 & 0,86 & 0,76 & 0,97 \\
\hline 65 e mais & 0,60 & 0,55 & 0,65 & 0,50 & 0,45 & 0,56 & 0,76 & 0,66 & 0,86 \\
\hline \multicolumn{10}{|c|}{ Escolaridade (anos) } \\
\hline 0 a 8 & 1,00 & & & 1,00 & & & 1,00 & & \\
\hline 9 a 11 & 1,48 & 1,37 & 1,58 & 1,60 & 1,45 & 1,77 & 1,32 & 1,19 & 1,46 \\
\hline 12 e mais & 1,83 & 1,70 & 1,96 & 1,83 & 1,66 & 2,03 & 1,85 & 1,68 & 2,05 \\
\hline \multicolumn{10}{|c|}{ Raça/cora } \\
\hline Branca & 1,00 & & & 1,00 & & & 1,00 & & \\
\hline Preta & 0,94 & 0,86 & 1,02 & 1,06 & 0,95 & 1,18 & 0,80 & 0,70 & 0,91 \\
\hline Parda & 0,98 & 0,93 & 1,03 & 1,07 & 1,00 & 1,15 & 0,87 & 0,81 & 0,94 \\
\hline \multicolumn{10}{|c|}{ Plano de saúde } \\
\hline Sim & 1,00 & & & 1,00 & & & 1,00 & & \\
\hline Não & 0,84 & 0,80 & 0,88 & 1,11 & 1,04 & 1,19 & 0,77 & 0,72 & 0,84 \\
\hline
\end{tabular}


Tabela 2. (cont.)

\begin{tabular}{|c|c|c|c|c|c|c|c|c|c|}
\hline \multicolumn{10}{|c|}{ Tabagismo } \\
\hline Não fumante & 1,00 & & & 1,00 & & & 1,00 & & \\
\hline Ex-fumante & 0,96 & 0,91 & 1,02 & 0,94 & 0,87 & 1,02 & 1,04 & 0,95 & 1,13 \\
\hline Fumante & 0,74 & 0,67 & 0,81 & 0,76 & 0,67 & 0,85 & 0,69 & 0,58 & 0,82 \\
\hline \multicolumn{10}{|c|}{ IMC } \\
\hline Normal & 1,00 & & & 1,00 & & & 1,00 & & \\
\hline Sobrepeso & 1,08 & 1,03 & 1,14 & 1,08 & 1,01 & 1,15 & 1,08 & 1,01 & 1,17 \\
\hline Obesidade & 0,88 & 0,82 & 0,95 & 0,87 & 0,79 & 0,96 & 0,89 & 0,80 & 0,99 \\
\hline \multicolumn{10}{|c|}{ Consumo recomendado de frutas e hortaliças ( 5 ou mais porções diárias) } \\
\hline $\operatorname{Sim}$ & 1,00 & & & 1,00 & & & 1,00 & & \\
\hline Não & 0,75 & 0,70 & 0,80 & 0,83 & 0,78 & 0,88 & 0,75 & 0,70 & 0,80 \\
\hline \multicolumn{10}{|c|}{ Consumo recomendado de frutas e hortaliças ( 5 ou mais dias por semana) } \\
\hline \multicolumn{10}{|l|}{$\operatorname{Sim}$} \\
\hline Não & 0,70 & 0,67 & 0,73 & 0,78 & 0,73 & 0,83 & 0,63 & 0,59 & 0,67 \\
\hline \multicolumn{10}{|c|}{ Consumo abusivo de bebidas alcoólicas } \\
\hline Não & 1,00 & & & 1,00 & & & 1,00 & & \\
\hline $\operatorname{Sim}$ & 1,05 & 1,00 & 1,12 & 1,03 & 0,96 & 1,10 & 1,13 & 1,02 & 1,25 \\
\hline \multicolumn{10}{|c|}{ Hipertensão } \\
\hline Não & 1,00 & & & 1,00 & & & 1,00 & & \\
\hline $\operatorname{Sim}$ & 0,95 & 0,89 & 1,01 & 0,97 & 0,89 & 1,06 & 0,92 & 0,84 & 1,00 \\
\hline \multicolumn{10}{|c|}{ Diabetes } \\
\hline Não & 1,00 & & & 1,00 & & & 1,00 & & \\
\hline $\operatorname{Sim}$ & 0,97 & 0,86 & 1,09 & 1,00 & 0,84 & 1,20 & 0,93 & 0,80 & 1,09 \\
\hline \multicolumn{10}{|c|}{ Colesterol } \\
\hline Não & 1,00 & & & 1,00 & & & 1,00 & & \\
\hline $\operatorname{Sim}$ & 1,01 & 0,95 & 1,07 & 0,94 & 0,87 & 1,03 & 1,06 & 0,98 & 1,14 \\
\hline \multicolumn{10}{|c|}{ № de doenças crônicas } \\
\hline 0 & 1,00 & & & 1,00 & & & 1,00 & & \\
\hline 1 & 0,97 & 0,92 & 1,03 & 0,95 & 0,88 & 1,03 & 1,00 & 0,92 & 1,08 \\
\hline 2 ou 3 & 0,97 & 0,89 & 1,07 & 0,96 & 0,84 & 1,10 & 0,96 & 0,85 & 1,08 \\
\hline
\end{tabular}

Fonte: Elaboração própria, com base em dados da Vigitel, 2013.

* RP ajustada por idade, sexo e escolaridade.

** RP ajustada por idade e escolaridade.

a Excluídas as categorias cor da pele amarela, vermelha, não sabe e não quis informar.

Nota: Percentual ponderado para ajustar a distribuição sociodemográfica da amostra Vigitel à distribuição da população adulta de cada cidade projetada para o ano de 2012.

\section{Discussão}

O estudo atual apontou que a prevalência da AFTL foi de cerca de um terço da população adulta. Ser mais jovem, do sexo masculino, com maior escolaridade, da cor branca, possuir plano de saúde, não fumar e consumir o recomendado de frutas e hortaliças aumentam a chance da prática da AFTL. Observou-se ainda que as pessoas eutróficas e as com sobrepeso são mais ativas, as pessoas com obesidade praticam menos AF. 
De acordo com o estudo de Malta et al. ${ }^{15}$, existe uma tendência de aumento no nível de AF nas capitais brasileiras, diferentemente da atividade de deslocamento, que se encontra com tendência de queda. Sabe-se que a AFTL confere maiores benefícios à saúde e às habilidades esportivas ${ }^{7}$. A OMS estabelece como meta reduzir o sedentarismo da população, o que resultaria em melhor qualidade de vida, considerando não apenas os efeitos biológicos e psíquicos como também os efeitos sociais ${ }^{8,16}$. Para tal, têm sido preconizados programas públicos de acesso e incentivo às práticas corporais regulares que podem melhorar a saúde da população e reduzir as DCNT ${ }^{3,7,17}$.

É conhecido que, com o processo de envelhecimento, ocorre um declínio nos níveis de AFTL ${ }^{15}$, conforme observado no atual estudo. Evidências têm demonstrado que crianças e adolescentes que praticam $\mathrm{AF}$ tendem a se tornar adultos ativos, o que contribuirá para o envelhecimento também ativo e saudável18.

Semelhante ao encontrado na presente pesquisa, Pan et al. ${ }^{19}$ demonstraram que quanto mais elevado é o nível de escolaridade, maiores serão as possiblidades, facilidades e acesso a equipamentos, a locais públicos e privados adequados e seguros para a prática da AFTL ${ }^{3,15}$, alertando para a necessidade de investir em espaços que incentivem a prática de $\mathrm{AF}$, acessíveis à população de baixa escolaridade e renda ${ }^{15,17}$.

Em relação à raça/cor, verificaram-se diferenças significativas para as mulheres negras e pardas, que apresentaram uma associação inversa com a AFTL, mesmo quando ajustado por idade e escolaridade. Esses achados foram também relatados em outros estudos, indicando uma desigualdade em relação à raça/cor nas oportunidades de prática de AF e o quanto algumas vulnerabilidades têm consequências sobre o estado de saúde das populações ${ }^{20}$.

No estudo atual, diferentemente dos homens, mulheres sem plano de saúde foram menos ativas. Malta e Bernal ${ }^{\mathbf{2 1}}$ apontam que ter plano de saúde está fortemente associado à maior escolaridade; assim como pode ser considerada a relação de um proxy de renda e maior escolaridade à maior prática de $\mathrm{AF}$. Torna-se importante elaborar políticas públicas que minimizem as iniquidades ao sexo feminino, oportunizando maior disponibilidade de tempo livre, engajamento nas AFTL e vivências corporais que favoreçam uma cultura do movimento e maior participação social ${ }^{20}$.

O presente estudo apontou uma associação inversa entre tabaco e AF, confirmando que o fumo se associa com maiores riscos à saúde 1,8,22 .

Os indivíduos eutróficos e os que apresentaram sobrepeso foram mais ativos; e, ao contrário, os obesos foram menos ativos. Os estudos demonstram que a prática de AF é benéfica em todas as idades, além de ser fundamental para o controle do peso, aliada a uma alimentação saudável2,22. Esses fatores podem motivar pessoas com sobrepeso a manter uma rotina de exercícios. Ao contrário, nem sempre é fácil incorporar a prática corporal à rotina diária do indivíduo com obesidade, pois, na maioria dos casos, eles possuem falta de condicionamento físico ${ }^{7}$. Torna-se necessária a construção de políticas públicas que priorizem estratégias e ações para o enfrentamento da obesidade e que atuem de forma integrada para mudanças de comportamento sobre os determinantes e condicionantes dessa doença, como, por exemplo, o Plano Nacional de Enfrentamento das Doenças Crônicas Não Transmissíveis, o 'Guia alimentar para a população brasileira' e o Programa Academia da Saúde 1,8,22,23.

O consumo recomendado de frutas e hortaliças, tanto diariamente quanto semanalmente, neste estudo, mostrou estar relacionado com a prática regular de $\mathrm{AF}$, o que tem sido confirmado por outros trabalhos. Dessa forma, aliar uma boa orientação de AF com uma alimentação saudável, principalmente de frutas e vegetais, torna-se uma das melhores apostas e estratégias para melhoria da saúde, para a redução da mortalidade, além de economia aos cofres públicos, devido ao custo-benefício ${ }^{22,23}$.

Estudos associaram o uso de bebida alcoólica de forma abusiva à inatividade física no tempo livre e a fatores de risco para as DCNT ${ }^{24}$, discordando do estudo atual. Além disso, o 
consumo analisado no Vigitel sobre beber de forma abusiva, em binge, é mais comum em pessoas mais jovens, mais propensas a essa prática. Entretanto, quando ajustado por idade, escolaridade e sexo, só se manteve significativo para as mulheres, podendo ser entendido em função de possíveis mudanças de comportamento das mulheres e de maior aproximação delas de práticas antes consideradas essencialmente masculinas ${ }^{25}$. Entretanto, recomenda-se novos estudos para confirmar esse achado.

As DCNT (hipertensão, diabetes, colesterol elevado e duas ou mais DCNT), após ajustes por idade, sexo e escolaridade, não apresentaram associação significativa com a AFTL, embora haja evidências descritas sobre o benefício da inclusão da AF no controle dessas doenças 18,22,23.

Dentre os limites do estudo, cita-se a avaliação da AF feita por meio de informação autorreferida, que poderia superestimar uma prática considerada desejável. Além disso, por se tratar de um estudo transversal, não é possível estabelecer uma relação de causa- efeito entre as variáveis. Por outro lado, vale salientar que a amostra é de tamanho suficiente para permitir a estratificação por sexo e possibilitar análises quanto à AFTL aqui detectadas. A variável aqui analisada, consumo de álcool abusivo, é mais frequente em populações jovens, podendo associar-se a essa faixa etária e aos comportamentos comuns nessa idade da vida, como a AFTL.

\section{Considerações finais}

Conclui-se que a AFTL se associa a fatores sociodemográficos, como ser mais jovem, homem, com elevada escolaridade, cor branca, com planos de saúde; e a hábitos saudáveis, tais como não fumar e consumir frutas e hortaliças. Dessa forma, torna-se relevante investir em políticas públicas de promoção à saúde e de redução de desigualdades em saúde. A OMS e o Ministério da Saúde fomentam estrategicamente programas e projetos, como as academias da saúde e o 'Guia alimentar da população brasileira', para o enfrentamento da pandemia do sedentarismo e a má alimentação. Tais estratégias possibilitam hábitos saudáveis, refletindo positivamente em oportunidades de inclusão social, equidade em saúde e aumento na expectativa de vida da população brasileira ${ }^{\mathbf{1}, \mathbf{4}, \mathbf{8}, \mathbf{2 2}, \mathbf{2 3} \text {. }}$

\section{Colaboradores}

Las Casas RCR, Bernal RTI, Jorge AO, Melo EM, Malta DC contribuíram substancialmente para a concepção, para o planejamento e para a análise dos dados descritos neste manuscrito; assim como para a elaboração do manuscrito e revisão crítica do conteúdo. Todos os autores aprovaram a versão final. 


\section{Referências}

1. Brasil. Ministério da Saúde. Secretaria de Vigilância em Saúde. Plano de ações estratégicas para enfrentamento das doenças crônicas não transmissíveis (DCNT) no Brasil 2011-2022. Brasília, DF: MS; 2011.

2. World Health Organization. Global Strategy on Diet, Physical Activity and Health. Geneva: WHO; 2004.

3. World Health Organization. Global status report on noncommunicable diseases 2014. Geneva: WHO; 2014.

4. Sallis JF, Cervero RB, Ascher W, et al. An ecological approach to creating active living communities. Annu Rev Public Health. 2006; 27:297-322.

5. Caspersen CJ, Powell KE, Christenson GM. Physical activity, exercise, and physical fitness: definitions and distinctions for health-related research. Public Health Rep. 1985; 100(2):126-131.

6. Nieman DC. Exercício e saúde. São Paulo: Manole; 1999.

7. World Health Organization. Global recommendations on physical activity for health. Geneva: WHO; 2010.

8. World Health Organization. Global Action Plan for the Prevention and Control of NCDs 2013-2020. Geneva: WHO; 2011.

9. United States of America. Department of Health and Human Services. Physical Activity Guidelines for Americans. Washington: HHS; 2008.

10. Lages SMRN, Emygdio RF, Monte ASI, et al. Motivation and self-esteem in university students' adherence to physical activity. Rev Salud Pública. 2015; 17(5):677-688.

11. Habermas J. Teoría de La Accion Comunicativa. Madrid: Taurus; 1987.

12. Brasil. Ministério da Saúde. Secretaria de Vigilância em Saúde. VIGITEL Brasil 2007: vigilância de fatores de risco e proteção para doenças crônicas por inquérito telefônico. Brasília, DF: MS; 2007.

13. Izrael D, Hoaglin DC, Battaglia MP. A SAS Macro for Balancing a Weighted Sample: Proceedings of the Twenty-Fifth Annual SAS Users Group International Conference. SAS Institute Inc. 2000; 275:1350-1355.

14. Stata Corporation. Stata Statistical Software: Release 12.1. Stata Corporation: College Station. Texas: Stata; 2012.

15. Malta DC, Andrade S, Santos M, et al. Tendências dos indicadores de atividade física em adultos: Conjunto de capitais do Brasil 2006-2013. Rev Bras Ativ Fís Saúde. 2015; 20(2):141-151.

16. Santos ALP, Simões AC. Educação Física e qualidade de vida: reflexões e perspectivas. Saúde Soc. 2012; 21(1):181-192.

17. Malta DC, Silva JB. Policies to promote physical activity in Brazil. Lancet. 2012; 380(9838):195-196.

18. Shah RV, Murthy VL, Colangelo LA, et al. Association of fitness in young adulthood with survival and cardiovascular risk: the Coronary Artery Risk Development in Young Adults (CARDIA) study. JAMA Intern Med. 2016; 176(1):87-95.

19. Pan SY, Cameron C, DesMeules M, et al. Individual, social, environmental, and physical environmental correlates with physical activity among Canadians: a cross-sectional study. BMC Public Health. 2009; 9(1):21-33.

20. Ray R. An intersectional analysis to explaining a lack of physical activity among middle class black women. Sociol Compass. 2014; 8(6):780-791.

21. Malta DC, Bernal RTI. Comparison of risk and protective factors for chronic diseases in the population with and without health insurance in the Brazilian capitals, 2011. Rev Bras Epidemiol. 2014; 17:241-255. 
22. Bloom DE, Chisholm D, Jané-Llopis E, et al. From burden to "best buys": reducing the economic impact of non-communicable disease in low-and middle-income countries. Geneva: WHO; 2011.

23. Lopes A, Ferreira A, Mendonça R, et al. Estratégia de promoção à saúde: programa academia da cidade de Belo Horizonte. Rev Bras Ativ Fís Saúde. 2016; 21(4):381-386.

24. Balbinot AD, Araujo RB, Alves GSL. Níveis de atividade física e uso de substâncias psicoativas em adolescentes escolares da região metropolitana de Por- to Alegre. Clinic Biomed Research. 2013; 33(3-4).

25. Machado ÍE, Lana FCF, Felisbino-Mendes MS, et al. Factors associated with alcohol intake and alcohol abuse among women in Belo Horizonte, Minas Gerais State, Brazil. Cad. Saúde Pública. 2013; 29(7):14491459.

Recebido em 30/04/2017

Aprovado em 24/11/2018

Conflito de interesses: inexistente

Suporte financeiro: não houve 